Article

\title{
Determining Fuzzy Distance between Sets by Application of Fixed Point Technique Using Weak Contractions and Fuzzy Geometric Notions
}

\author{
Parbati Saha ${ }^{1}$, Shantau Guria ${ }^{1}$, Binayak S. Choudhury ${ }^{1}$ and Manuel De la Sen ${ }^{2, *(D)}$ \\ 1 Department of Mathematics, Indian Institute of Engineering Science and Technology, Shibpur, \\ Howrah 711103, West Bengal, India; parbati_saha@yahoo.co.in (P.S.); sguria.math@gmail.com (S.G.); \\ binayak12@yahoo.co.in (B.S.C.) \\ 2 Institute of Research and Development of Processes IIDP, Faculty of Science and Technology, \\ University of the Basque Country, PO Box 644, de Bilbao, Barrio Sarriena, 48940 Leioa, Bizkaia, Spain \\ * Correspondence: manuel.delasen@ehu.eus
}

Received: 4 June 2019; Accepted: 18 June 2019; Published: 19 June 2019

\begin{abstract}
In the present paper, we solve the problem of determining the fuzzy distance between two subsets of a fuzzy metric space. We address the problem by reducing it to the problem of finding an optimal approximate solution of a fixed point equation. This approach is well studied for the corresponding problem in metric spaces and is known as proximity point problem. We employ fuzzy weak contractions for that purpose. Fuzzy weak contraction is a recently introduced concept intermediate to a fuzzy contraction and a fuzzy non-expansive mapping. Fuzzy versions of some geometric properties essentially belonging to Hilbert spaces are considered in the main theorem. We include an illustrative example and two corollaries, one of which comes from a well-known fixed point theorem. The illustrative example shows that the main theorem properly includes its corollaries. The work is in the domain of fuzzy global optimization by use of fixed point methods.
\end{abstract}

Keywords: fuzzy metric space; global optimization; proximity point; weak contraction; fuzzy P-property

\section{Introduction and Mathematical Preliminaries}

Fixed point methods in mathematics are well known for their potentials of applications. There are several uses of the Banach contraction mapping principle itself. Over the years, the idea of contraction was generalized and extended in several directions. Other types of contractive mappings having very different features like discontinuity, etc. have also appeared in a large way in the context of fixed point theory. Books like [1-3] provide an account of this line of study along with applications that are experiencing rapid expansion even today.

A new tenet in mathematics appeared with the introduction of fuzzy set theory by Zadeh [4] in 1965 which made quick headways in different domains of mathematics and its application areas. This is also the case with functional analysis. In particular, a fuzzy version of metric space appeared in the work of Kramosil et al. [5] which was further modified by George et al. [6]. In this modified definition, the topology is a Hausdorff topology. Fixed point theory in this space has developed in paralell with that which is in the ordinary metric space. Today, fuzzy fixed point theory is a subject by itself regarding the multifacet development that it has undergone. Some instances of these works are [7-11].

Here, we concentrate on an application of a weak contractive mapping for obtaining the distance between two appropriate subsets of a fuzzy metric space. The problem is formally known as proximity point problem and is solved by the methodology of fixed point theory. 
The discussion in this paper is based on notions that are specific for the purpose of studying the structure of fuzzy metric spaces rather than those used for the most general description of the fuzzy systems [12].

In metric space, the proximity point problem is well studied and is briefly described in the following.

Let $(X, d)$ be a metric space, $A, B$ be two nonempty subsets of $X$ and $f: A \rightarrow B$ be a mapping. The distance between the sets $A$ and $B$ is $d(A, B)=\inf \{d(a, b): a \in A, b \in B\}$. It is realized by finding a point $x^{*} \in A$ such that $d\left(x^{*}, f x^{*}\right)=d(A, B)$ in which case $x^{*}$ is called a best proximity point of $f$. There can be more than one such point in general. The problem is essentially a global optimization problem that admits a fixed point approach. Here, our goal is to find some $p \in A$ at which $d(x, T x)$ has a minimum for $x$ varying over $A$ which is such that the global minimum $d(A, B)$ is attained at $z$, that is, where $d(p, T p)=d(A, B)$. The point $p$ is a fixed point in a special case where $A \cap B \neq \phi$. In that sense, the above concept is an extension of the idea of fixed point. We can apply fixed point methodologies to the proximity point problem by converting it to a problem of obtaining a global optimal approximate solution of the equation $T x=x$. It should be noted that the exact solution of the equation mentioned above may not exist in the general case, which is when $A \cap B=\phi$. This is actually the case in which we are interested in this work. Our main theorem is proved without any assumption on the intersection of $A$ and $B$. Moreover, an illustrative example supports the case where $A$ and $B$ are disjointed. Contraction mappings of various types are employed in these problems. Several works on this topic are noted in [13-19].

The corresponding problem in the fuzzy metric space was considered in recent works like [20-23]. As in the case of the problem in metric spaces, it should be possible to solve the problem by applying contractions of various types. We apply a fuzzy weak contraction for the above purpose. In this context, various techniques of fuzzy approximations may be noted that have been developed in recent times, for which we refer to the book of Anastassiou et al. [24] and references therein. Here, we find an optimal approximate solution of the fixed point equation in the fuzzy sense by fixed point methods.

It is well known that fixed point techniques are considered as strong methods in the problem of applied mathematics. Due to its flexibility and versatility, fuzzy metric spaces are appropriate structures for modelling many real world problems in which there are inbuilt uncertainties. An instance is the work of Gregori et al. [25] where a problem of colour recognition has been addressed.

In an effort to generalize the Banach contraction, a special type of mapping that is intermediate between a contraction and a non-expansive mapping was introduced in the context of Hilbert spaces by Alber et al. [26]. The definition was adapted to metric spaces in the work of Rhoades [27] in which a weak contraction mapping principle was established. The definition of weak contraction in metric space is as follows.

Let $(X, d)$ be a metric space. A map $T$ of $X$ to itself is called a weak contraction, if for each $x, y \in X$,

$$
d(T x, T y) \leq d(x, y)-\phi(d(x, y)),
$$

where $\phi:[0, \infty) \rightarrow[0, \infty)$ is nondecreasing and continuous such that $\phi(t)>0$ for all $t>0, \phi(0)=0$, and $\lim _{t \rightarrow \infty} \phi(t)=\infty$.

Rhoades [27] has shown that weak contractions as defined above have unique fixed points in a complete metric space. Further generalizations and extensions of the idea mentioned above have appeared in research works [28-30]. In fuzzy metric spaces, weak contraction was introduced by Saha et al. [31].

For all purposes in this paper, by a fuzzy metric space, we mean that which is given in the following definition, which is due to George et al. [6].

Definition 1 ([6]). A fuzzy metric space is a 3-tuple $(X, M, *)$, where $X$ is an arbitrary non-empty set, $M$ is a fuzzy set on $X^{2} \times(0, \infty)$ such that for all $x, y, z \in X$ and $t, s>0$ : 
1. $M(x, y, t)>0$,

2. $M(x, y, t)=1 \Longleftrightarrow x=y$,

3. $M(x, y, t)=M(y, x, t)$,

4. $M(x, y, t) * M(y, z, s) \leq M(x, z, t+s)$, and

5. $M(x, y,):.(0, \infty) \longrightarrow(0,1]$ is continuous,

where $*$ is a continuous $t$-norm. We recall that a t-norm is a function $*:[0,1]^{2} \rightarrow[0,1]$ for which

1. $a * b=b * a$ for all $a, b \in[0,1]$,

2. $a *(b * c)=(a * b) * c$ for all $a, b, c \in[0,1]$,

3. $a * 1=a$ for all $a \in[0,1]$,

4. $a * b \leq c * d$ whenever $a \leq c$ and $b \leq d$, for each $a, b, c, d \in[0,1]$.

The topology on the space $(X, M, *)$ is that which is generated by the open balls

$$
B(x, \lambda, s)=\{y \in X: M(x, y, s)>1-\lambda\}, \text { where } s>0 \text { and } 0<\lambda<1 .
$$

The topology is a Hausdorff topology [6]. Actually, Definition 1 is introduced by modifying the definition given in [5] for the purpose of ensuring that the space satisfies the $T_{2}$-axiom.

From a topological viewpoint, the above property of the space is the reason for our consideration of the notion of fuzzy metric space as given in Definition 1. In fact, this is one of the reasons for the vast development of fixed point results and methodologies in the framework of this space.

Definition 2 ([6]). A sequence $\left\{x_{n}\right\}$ in a fuzzy metric space $(X, M, *)$ is said to be convergent to a point $x$ in $X$ if $\lim _{n \rightarrow \infty} M\left(x_{n}, x, s\right)=1$ for all $s>0$.

Definition 3 ([6]). A sequence $\left\{x_{n}\right\}$ in a fuzzy metric space $(X, M, *)$ is a Cauchy sequence if, for each $\varepsilon$ with $0<\varepsilon<1$ and $t>0$, we can find a positive integer $N$ such that $M\left(x_{n}, x_{m}, t\right)>1-\varepsilon$ for each $n, m \geq N$.

If every Cauchy sequence is convergent, then the fuzzy metric space is said to be complete.

The following lemma that appears in [8] for fuzzy metric spaces defined by Kramosil et al. [5] is also true for the fuzzy metric space given by Definition 1 .

Lemma 1 ([8]). Let $(X, M, *)$ be a fuzzy metric space. Then, for fixed $x, y \in X, M(x, y,$.$) is non-decreasing$ in the third variable.

Lemma 2 ([32]). $M$ is continuous on $X^{2} \times(0, \infty)$.

In the following, we define non-self weak contraction.

Definition 4. Let $A, B$ be two subsets of $X$, where $(X, M, *)$ is a complete fuzzy metric space. Let $f: A \rightarrow B$ be a mapping that satisfies the following inequality:

$$
\psi(M(f x, f y, t)) \leq \psi(M(x, y, t))-\phi(M(x, y, t)),
$$

where $x, y \in A, t>0$ and $\psi, \phi:(0,1] \rightarrow[0, \infty)$ are such that,

(i) $\psi$ is monotone decreasing and continuous with $\psi(t)=0 \Longleftrightarrow t=1$,

(ii) $\phi$ is lower semi continuous with $\phi(s)=0 \Longleftrightarrow s=1$.

Then, we call $f$ a non-self weak contraction from $A$ to $B$.

In the special case where $A=B$, the above definition reduces to that of weak contraction introduced in [31]. It is shown in [31] that weak contraction is weaker that a fuzzy Banach contraction 
but stronger than a fuzzy non-expensive mapping. Our special interest is in the most general case where $A$ and $B$ are disjoint. The inequality (2) can be seen to be comparable in form to Label (1) although the contexts are different.

Definition 5 ([22]). Let $(X, M, *)$ be a fuzzy metric space. The fuzzy distance of a point $x \in X$ from a nonempty subset $A$ of $X$ is

$$
M(x, A, t)=\sup _{a \in A} M(x, a, t) \text { for all } t>0
$$

and the fuzzy distance between two nonempty subsets $A$ and $B$ of $X$ is

$$
M(A, B, t)=\sup \{M(a, b, t): a \in A, b \in B\} \text { for all } t>0 .
$$

Let $A$ and $B$ be two disjoint nonempty subsets of a fuzzy metric space $(X, M, *)$. We write

$$
\begin{aligned}
& A_{0}=\{x \in A: \exists y \in \text { Bsuchthat } M(x, y, t)=M(A, B, t) \text { forallt }>0\}, \\
& B_{0}=\{y \in B: \exists x \in \text { Asuchthat } M(x, y, t)=M(A, B, t) \text { forallt }>0\} .
\end{aligned}
$$

Note: Analogous to the above definition, there are notions of $A_{0}(t)$ and $B_{0}(t)$ that have been used in fuzzy proximity point problems in work $[20,33]$. The difference with the above definition is that they are independent from the parameter $t$ here.

Definition 6 ([22,23]). Let $A, B$ be two non-empty subsets of $X$ where $(X, M, *)$ be a fuzzy metric space. An element $x^{*} \in A$ is defined as a fuzzy best proximity point of the mapping $f: A \rightarrow B$ if it satisfies the condition that for all $t>0$

$$
M\left(x^{*}, f x^{*}, t\right)=M(A, B, t) .
$$

In the following, we describe a property of pair of subsets of a fuzzy metric space. It is essentially a geometric property.

Definition 7 ([21]). Let $(A, B)$ be a pair of disjoint nonempty subsets of $X$ where $(X, M, *)$ is a fuzzy metric space. Then, the pair $(A, B)$ is said to satisfy the fuzzy P-property if, for all $t>0$ and $x_{1}, x_{2} \in A, y_{1}, y_{2} \in B$,

$$
M\left(x_{1}, y_{1}, t\right)=M(A, B, t) \text { and } M\left(x_{2}, y_{2}, t\right)=M(A, B, t)
$$

jointly imply that

$$
M\left(x_{1}, x_{2}, t\right)=M\left(y_{1}, y_{2}, t\right) .
$$

The $P$-property is a geometric property that is automatically valid in Hilbert spaces for non-empty closed and convex pairs of sets [18], but does not hold in arbitrary Banach spaces. In metric spaces, the $P$-property for pairs of subsets is separately assumed for specific purposes $[15,18,34]$. The above definition is a fuzzy extension of that.

We state the following lemmas that are used subsequently.

Lemma 3 ([31]). If $*$ is a continuous $t$-norm, and $\left\{\alpha_{n}\right\},\left\{\beta_{n}\right\}$ and $\left\{\gamma_{n}\right\}$ are sequences in $[0,1]$ for which $\alpha_{n} \rightarrow \alpha, \gamma_{n} \rightarrow \gamma$ as $n \rightarrow \infty$, then $\varlimsup_{k \rightarrow \infty}\left(\alpha_{k} * \beta_{k} * \gamma_{k}\right)=\alpha * \varlimsup_{k \rightarrow \infty} \beta_{k} * \gamma$ and

$\varliminf_{k \rightarrow \infty}\left(\alpha_{k} * \beta_{k} * \gamma_{k}\right)=\alpha * \varliminf_{k \rightarrow \infty} \beta_{k} * \gamma$.

Lemma 4 ([31]). Let a sequence of functions $\{f(n,):.[0, \infty) \rightarrow[0,1], n=0,1,2, \ldots . .$.$\} be such that f(n,$.$) is$ monotone increasing and continuous for each $n \geq 0$. Then, $\varlimsup_{n \rightarrow \infty} f(n, t)$ is a left continuous function in $t$ and $\varliminf_{n \rightarrow \infty} f(n, t)$ is a right continuous function in $t$. 


\section{Main Results}

Theorem 1. Let $(X, M, *)$ be a complete fuzzy metric space. Let $A$ and $B$ be two closed subsets of $X$ and $f: A \rightarrow B$ be a non-self weak contraction mapping such that the following conditions are satisfied:

(i) $(A, B)$ satisfies the fuzzy P-property,

(ii) $f\left(A_{0}\right) \subseteq B_{0}$,

(iii) $A_{0}$ is nonempty.

Then, there exists an element $x^{*} \in A$ which is a fuzzy best proximity point of $f$.

Proof of Theorem 1. By assumption (iii), $A_{0}$ is nonempty. Let $x_{0} \in A_{0}$. Since $f\left(A_{0}\right) \subseteq B_{0}$, there exists $x_{1} \in A_{0}$ such that

$$
M\left(x_{1}, f x_{0}, t\right)=M(A, B, t) \text { for all } t>0 .
$$

Again, since $f\left(A_{0}\right) \subseteq B_{0}$, there exists $x_{2} \in A_{0}$ such that

$$
M\left(x_{2}, f x_{1}, t\right)=M(A, B, t) \text { for all } t>0 .
$$

By repeating the above procedure, we obtain a sequence $\left\{x_{n}\right\}$ in $A_{0}$ such that, for all $n \geq 1$, for all $t>0$

$$
M\left(x_{n}, f x_{n-1}, t\right)=M(A, B, t)
$$

In addition, we can write the above as

$$
M\left(x_{n+1}, f x_{n}, t\right)=M(A, B, t) \text { for all } n \geq 0, \text { for all } t>0
$$

Since fuzzy P-property holds for the pair $(A, B)$, from (3) and (4), for each $n \geq 1, t>0$, we obtain

$$
M\left(x_{n}, x_{n+1}, t\right)=M\left(f x_{n-1}, f x_{n}, t\right) .
$$

Putting $x=x_{n-1}$ and $y=x_{n}$ in (2) we obtain, for all $n \geq 1, t>0$,

$$
\psi\left(M\left(f x_{n-1}, f x_{n}, t\right)\right) \leq \psi\left(M\left(x_{n-1}, x_{n}, t\right)\right)-\phi\left(M\left(x_{n-1}, x_{n}, t\right)\right)
$$

Using (5) and (6), we have

$$
\psi\left(M\left(x_{n}, x_{n+1}, t\right)\right) \leq \psi\left(M\left(x_{n-1}, x_{n}, t\right)\right)-\phi\left(M\left(x_{n-1}, x_{n}, t\right)\right)
$$

It follows from the inequality obtained above that $\psi\left(M\left(x_{n}, x_{n+1}, t\right) \leq \psi\left(M\left(x_{n-1}, x_{n}, t\right)\right.\right.$ where $n \geq 1, t>0$. Since $\psi$ is monotone decreasing, we have that

$M\left(x_{n}, x_{n+1}, t\right) \geq M\left(x_{n-1}, x_{n}, t\right)$, that is, $\left\{M\left(x_{n}, x_{n+1}, t\right)\right\}$ is a monotone increasing sequence in $[0,1]$. Therefore, for each choice of $t>0$, there exists $a(t)$ such that

$$
\lim _{n \rightarrow \infty} M\left(x_{n}, x_{n+1}, t\right)=a(t) \leq 1
$$

Now, taking $n \rightarrow \infty$ in (7), using continuity of $\psi$ and lower semi-continuity of $\phi$, we obtain, for all $t>0, \psi(a(t)) \leq \psi(a(t))-\phi(a(t))$, which implies that $a(t)=1$, for all $t>0$.

From the above, it follows that, for all $t>0$,

$$
\lim _{n \rightarrow \infty} M\left(x_{n}, x_{n+1}, t\right)=1
$$

Next, we establish that $\left\{x_{n}\right\}$ is a Cauchy sequence in $A$. We suppose, on the contrary, that $\left\{x_{n}\right\}$ is not a Cauchy sequence in $A$. Then, Definition 3 is not satisfied by the sequence $\left\{x_{n}\right\}$ and, therefore, 
there exists some $\epsilon>0$ and some $\lambda$ with $0<\lambda<1$, for which it is possible to find two subsequences $\left\{x_{m(k)}\right\}$ and $\left\{x_{n(k)}\right\}$ of $\left\{x_{n}\right\}$ with

$$
n(k)>m(k)>k
$$

such that

$$
M\left(x_{m(k)}, x_{n(k)}, \epsilon\right) \leq(1-\lambda)
$$

for all positive integer $\mathrm{k}$.

Let $n(k)$ be the smallest integer that exceeds $m(k)$ for which (10) holds. Then, for every positive integer $k$,

$$
M\left(x_{m(k)}, x_{n(k)-1}, \epsilon\right)>(1-\lambda) .
$$

Then, for all $k \geq 1,0<s<\frac{\epsilon}{2}$, we obtain that

$$
\begin{aligned}
(1-\lambda) & \geq M\left(x_{m(k)}, x_{n(k)}, \epsilon\right) \\
& \geq M\left(x_{m(k)}, x_{m(k)-1}, s\right) * M\left(x_{m(k)-1}, x_{n(k)-1}, \epsilon-2 s\right) \\
& * M\left(x_{n(k)-1}, x_{n(k)}, s\right) .
\end{aligned}
$$

For all $t>0$, we denote

$$
h_{1}(t)=\varlimsup_{k \rightarrow \infty} M\left(x_{m(k)-1}, x_{n(k)-1}, t\right) .
$$

Taking the limit supremum on both sides of (12), using (9), the properties of $M$ and *, by Lemma (3), we obtain

$$
(1-\lambda) \geq 1 * \varlimsup_{k \rightarrow \infty} M\left(x_{m(k)-1}, x_{n(k)-1}, \epsilon-2 s\right) * 1=h_{1}(\epsilon-2 s) .
$$

Since $\mathrm{M}$ is continuous and monotone increasing in $t$ (by Lemma 1), it follows by an application of Lemma 4 that $h_{1}$, as given in (13), is continuous from the left. In view of the above, letting $s \rightarrow 0$ in (14), it then follows that

$$
\varlimsup_{k \rightarrow \infty} M\left(x_{m(k)-1}, x_{n(k)-1}, \epsilon\right) \leq(1-\lambda) .
$$

Let

$$
h_{2}(t)=\varliminf_{k \rightarrow \infty} M\left(x_{m(k)-1}, x_{n(k)-1}, t\right), t>0 .
$$

Again, for all $k \geq 1, s>0$,

$$
\begin{aligned}
M\left(x_{m(k)-1}, x_{n(k)-1}, \epsilon+s\right) & \geq M\left(x_{m(k)-1}, x_{m(k)}, s\right) * M\left(x_{m(k)}, x_{n(k)-1}, \epsilon\right) \\
& \geq M\left(x_{m(k)-1}, x_{m(k)}, s\right) *(1-\lambda),(b y(11)) .
\end{aligned}
$$

Taking limit infimum as $k \rightarrow \infty$ in (17), due to (9), it follows that

$$
\begin{aligned}
h_{2}(\epsilon+s)=\varliminf_{k \rightarrow \infty} M\left(x_{m(k)-1}, x_{n(k)-1}, \epsilon+s\right) & \geq \varliminf_{k \rightarrow \infty} M\left(x_{m(k)-1}, x_{m(k)}, s\right) *(1-\lambda) \\
& =1 *(1-\lambda)=(1-\lambda) .
\end{aligned}
$$


Since $\mathrm{M}$ is continuous and by monotone increasing in $t$ (by Lemma 1 ), it follows by an application of Lemma 4 that $h_{2}$, as given in (16) is continuous from the right.

In view of the above, letting $s \rightarrow 0$ in (18), it then follows that

$$
\varliminf_{k \rightarrow \infty} M\left(x_{m(k)-1}, x_{n(k)-1}, \epsilon\right) \geq(1-\lambda) .
$$

Then, (15) and (19) imply that

$$
\lim _{k \rightarrow \infty} M\left(x_{m(k)-1}, x_{n(k)-1}, \epsilon\right)=(1-\lambda) .
$$

Again, by (10),

$$
\varlimsup_{k \rightarrow \infty} M\left(x_{m(k)}, x_{n(k)}, \epsilon\right) \leq(1-\lambda) .
$$

In addition, for all $k \geq 1, s>0$, we have

$$
M\left(x_{m(k)}, x_{n(k)}, \epsilon+2 s\right) \geq M\left(x_{m(k)}, x_{m(k)-1}, s\right) * M\left(x_{m(k)-1}, x_{n(k)-1}, \epsilon\right) * M\left(x_{n(k)-1}, x_{n(k)}, s\right) .
$$

Taking limit infimum as $k \rightarrow \infty$, using (9), (20) by Lemma 3, we obtain

$$
\varliminf_{k \rightarrow \infty} M\left(x_{m(k)}, x_{n(k)}, \epsilon+2 s\right) \geq 1 * \varliminf_{k \rightarrow \infty} M\left(x_{m(k)-1}, x_{n(k)-1}, \epsilon\right) * 1=1-\lambda .
$$

Since $\mathrm{M}$ is continuous and monotone increasing in $t$ (by Lemma 1), it follows from Lemma 4 that $\varliminf_{k \rightarrow \infty} M\left(x_{m(k)}, x_{n(k)}, t\right)$ is a right continuous function of $t$.

Taking $s \rightarrow 0$ in the above inequality, by Lemma 4 , we obtain

$$
\varliminf_{k \rightarrow \infty} M\left(x_{m(k)}, x_{n(k)}, \epsilon\right) \geq(1-\lambda) .
$$

Combining (21) and (22), we obtain

$$
\lim _{k \rightarrow \infty} M\left(x_{m(k)}, x_{n(k)}, \epsilon\right)=(1-\lambda)
$$

Again, from (4), we have

$$
M\left(x_{m(k)}, f x_{m(k)-1}, t\right)=M(A, B, t)
$$

and

$$
M\left(x_{n(k)}, f x_{n(k)-1}, t\right)=M(A, B, t) .
$$

Since $(A, B)$ satisfies the fuzzy P-property, we get from (24) and (25), for all $t>0$,

$$
M\left(x_{m(k)}, x_{n(k)}, t\right)=M\left(f x_{m(k)-1}, f x_{n(k)-1}, t\right) .
$$

Putting $x=x_{m(k)-1}, y=x_{n(k)-1}, t=\epsilon$ in (2) and using the result of (26), we obtain

$$
\begin{aligned}
\psi\left(M\left(x_{m(k)}, x_{n(k)}, \epsilon\right)\right) & =\psi\left(M\left(f x_{m(k)-1}, f x_{n(k)-1}, \epsilon\right)\right) \\
& \leq \psi\left(M\left(x_{m(k)-1}, x_{n(k)-1}, \epsilon\right)\right)-\phi\left(M\left(x_{m(k)-1}, x_{n(k)-1}, \epsilon\right)\right) .
\end{aligned}
$$


Letting $k \rightarrow \infty$ in the above inequality, using (20), (23), continuity of $\psi$ and the lower semi-continuity of $\phi$, we obtain

$$
\psi(1-\lambda) \leq \psi(1-\lambda)-\phi(1-\lambda)
$$

which is a contradiction since $\phi(1-\lambda) \neq 0$.

This shows that $\left\{x_{n}\right\}$ is a Cauchy sequence. Again, $(X, M, *)$ is complete. Therefore, there exists $x^{*} \in A$ for which

$$
\lim _{n \rightarrow \infty} x_{n}=x^{*} .
$$

Since $f$ is a weak contraction, then, for all $n \geq 1, t>0$,

$$
\psi\left(M\left(f x_{n}, f x^{*}, t\right)\right) \leq \psi\left(M\left(x_{n}, x^{*}, t\right)\right)-\phi\left(M\left(x_{n}, x^{*}, t\right)\right) .
$$

Letting $n \rightarrow \infty$, using continuity of $\psi$ and lower semi-continuity of $\phi$, we obtain

$$
\begin{aligned}
\lim _{n \rightarrow \infty} \psi\left(M\left(f x_{n}, f x^{*}, t\right)\right) & \leq \psi(1)-\phi(1) \\
& =0 .
\end{aligned}
$$

That is, using a property of $\psi$, for all $t>0$,

$$
\lim _{n \rightarrow \infty} M\left(f x_{n}, f x^{*}, t\right)=1,
$$

which implies that

$$
f x_{n} \rightarrow f x^{*} \text { as } n \rightarrow \infty .
$$

Taking limit $n \rightarrow \infty$ on both sides of equation (4), we get

$$
\lim _{n \rightarrow \infty} M\left(x_{n+1}, f x_{n}, t\right)=M(A, B, t) .
$$

Using the result of (27), (29) and applying Lemma 2, from (30) we have, for all $t>0$,

$$
M\left(x^{*}, f x^{*}, t\right)=M(A, B, t) .
$$

The proof of the theorem is thereby completed.

We have the following corollary of our theorem, which is a special case of the main theorem of [31] without the condition of partial order.

Corollary 1 ([31]). Let $f: X \rightarrow X$, where $(X, M, *)$ is a complete fuzzy metric space, and a mapping which satisfies the following inequality:

$$
\psi(M(f x, f y, t)) \leq \psi(M(x, y, t))-\phi(M(x, y, t)),
$$

where $x, y \in X, t>0$ and $\psi, \phi:(0,1] \rightarrow[0, \infty)$ are such that

(i) $\psi$ is monotone and continuous decreasing with $\psi(t)=0 \Longleftrightarrow t=1$,

(ii) $\phi$ is lower semi continuous with $\phi(s)=0 \Longleftrightarrow s=1$.

Then, $f$ has a fixed point in $X$. 
Proof of Corollary 1. The proof of the corollary follows by assuming $A=B=X$ in the above theorem. The P-property is not relevant to this corollary and is omitted.

We have the following well-known result due to Gregori and Sapena [9], which follows directly from the above corollary.

Corollary 2. (Fuzzy Banach Contraction mapping Principle [9])

Let $(X, M, *)$ be a complete fuzzy metric space and let $f: X \rightarrow X$ be such that

$$
\left(\frac{1}{M(f x, f y, t)}-1\right) \leq k\left(\frac{1}{M(x, y, t)}-1\right),
$$

where $x, y \in X, t>0$ and $k \in(0,1)$.

Then, $f$ has a fixed point.

Proof of Corollary 2. Let $\psi(s)=\frac{1-s}{s}$ and $\phi(s)=\frac{(1-k)(1-s)}{s}$, where $0<s \leq 1$. Then, we see that (32) implies (2), where $x, y \in X$ and $t>0$.

\section{Illustration}

In this section, we demonstrate an application of our theorem to an example. The illustrative example shows that Corollaries 1 and 2, proved independently in the literature, are in fact corollaries of the current Theorem 1 as claimed.

Example 1. Suppose that $X=\mathbb{R}^{2}$ with fuzzy metric

$$
M\left((x, y),\left(x^{\prime}, y^{\prime}\right), t\right)=\frac{t}{t+\left|x-x^{\prime}\right|+\left|y-y^{\prime}\right|} .
$$

Let $a * b=\min \{a, b\}$.

Consider two closed subsets of $X$,

$$
\begin{aligned}
& A=\{(0, x): 0 \leq x<\infty\}, \\
& B=\{(1, x): 0 \leq x<\infty\} .
\end{aligned}
$$

Let $\bar{x}, \bar{y} \in A$, where $\bar{x}=(0, x), \bar{y}=(0, y), x, y \geq 0$.

Let $h: A \rightarrow B$ be the mapping defined by

$$
h \bar{x}=\left(1, \ln \left(1+\frac{x}{2}\right)\right) .
$$

Here, we take two functions $\psi, \phi:(0,1] \rightarrow[0, \infty)$ given by

$$
\psi(a)=\frac{1-a}{a} \text { and } \phi(a)=\frac{1-a}{2 a} .
$$

Now, we verify fuzzy P- property.

Here, $M(A, B, t)=\frac{t}{1+t}$ for all $t>0$.

Here, $A_{0}=A$ and $B_{0}=B$ and $f\left(A_{0}\right) \subseteq B_{0}$.

Let $u_{1}=\left(0, x_{1}\right), u_{2}=\left(0, x_{2}\right) \in A$ and $v_{1}=\left(1, y_{1}\right), v_{2}=\left(1, y_{2}\right) \in B$ with

$$
M\left(u_{1}, v_{1}, t\right)=M(A, B, t) \text { for all } t>0
$$

and

$$
M\left(u_{2}, v_{2}, t\right)=M(A, B, t) \text { for all } t>0 .
$$


From (33), we get for all $t>0$,

$$
\frac{t}{t+1+\left|x_{1}-y_{1}\right|}=\frac{t}{t+1}
$$

which implies that $x_{1}=y_{1}$.

Similarly from (34), we get for all $t>0$,

$$
x_{2}=y_{2}
$$

Therefore, for all $t>0$,

$$
M\left(u_{1}, u_{2}, t\right)=\frac{t}{t+\left|x_{1}-x_{2}\right|}=\frac{t}{t+\left|y_{1}-y_{2}\right|}=M\left(v_{1}, v_{2}, t\right) .
$$

Now, for all $t>0$,

$$
\begin{aligned}
\psi(M(h \bar{x}, h \bar{y}, t)) & =\psi\left(M\left(\left(1, \ln \left(1+\frac{x}{2}\right)\right),\left(1, \ln \left(1+\frac{y}{2}\right)\right), t\right)\right) \\
& =\psi\left(\frac{t}{t+\left|\ln \left(1+\frac{x}{2}\right)-\ln \left(1+\frac{y}{2}\right)\right|}\right) \\
& =\frac{\left|\ln \left(1+\frac{x}{2}\right)-\ln \left(1+\frac{y}{2}\right)\right|}{t} .
\end{aligned}
$$

We have the following cases:

Case I $x \geq y$.

$$
\begin{aligned}
\frac{\left|\ln \left(1+\frac{x}{2}\right)-\ln \left(1+\frac{y}{2}\right)\right|}{t} & =\frac{\ln \left(\frac{1+\frac{x}{2}}{1+\frac{y}{2}}\right)}{t} \\
& =\frac{\ln \left(1+\frac{\frac{x-y}{2}}{1+\frac{y}{2}}\right)}{t} \\
& \leq \frac{\ln \left(1+\frac{x-y}{2}\right)}{t}, \text { as } \ln (1+x) \text { is an increasing function } \\
& \leq \frac{x-y}{2 t} \\
& \leq \frac{|x-y|}{2 t} \\
& \leq \frac{A}{2 t}, \text { where } A=|x-y| .
\end{aligned}
$$


Case II $x<y$.

$$
\begin{aligned}
\frac{\left|\ln \left(1+\frac{x}{2}\right)-\ln \left(1+\frac{y}{2}\right)\right|}{t} & =\frac{\ln \left(\frac{1+\frac{y}{2}}{1+\frac{x}{2}}\right)}{t} \\
& =\frac{\left.\ln \left(1+\frac{y-x}{2}\right) \frac{x}{2}\right)}{t} \\
& \leq \frac{\ln \left(1+\frac{y-x}{2}\right)}{t}, \text { as } \ln (1+x) \text { is an increasing function. } \\
& \leq \frac{y-x}{2 t} \\
& \leq \frac{|y-x|}{2 t} \\
& \leq \frac{A}{2 t} .
\end{aligned}
$$

Therefore, $\psi(M(h \bar{x}, h \bar{y}, t)) \leq \frac{A}{2 t}$, where $A=|x-y|$.

Now,

$$
\begin{aligned}
\psi(M(\bar{x}, \bar{y}, t))-\phi(M(\bar{x}, \bar{y}, t)) & =\psi\left(\frac{t}{t+|x-y|}\right)-\phi\left(\frac{t}{t+|x-y|}\right) \\
& =\frac{A}{t}-\frac{A}{2 t} \\
& =\frac{A}{2 t} \text {, where } A=|x-y| .
\end{aligned}
$$

Hence, for all $t>0$,

$$
\psi(M(h \bar{x}, h \bar{y}, t)) \leq \psi(M(\bar{x}, \bar{y}, t))-\phi(M(\bar{x}, \bar{y}, t)) .
$$

Thus, all the conditions of the Theorem 1 are satisfied from which it follows that $h$ has a fuzzy best proximity point. It is observed that $(0,0)$ is such point.

Note: The above illustration indicates that the result in Theorem 1 is an effective generalization of its corollaries, since the corollaries are not applicable to the above example. In particular, the main result actually contains the fuzzy Banach contraction mapping principle given by Gregori and Sapena [9] in complete fuzzy metric spaces in the form given in Corollary 2. For some interesting examples, we refer to [9].

\section{Conclusions}

The task of finding an optimized distance between two objects is considered to be an important problem of mathematics, which is at the root of several studies related to fields like geometry [35], mechanics [36], optimization [37], etc. Here, our candidates are sets and the framework of study is the general structure of fuzzy metric spaces. The prospects of the present study lie in the possibilities of further generalizations as well as in its possible applications in the domains of fuzzy geometry, fuzzy optimization, etc. The present paper is an instance from the recently developing area of fuzzy fixed point methodologies.

Author Contributions: All authors contributed equally and significantly in writing this article. All authors read and approved the final manuscript.

Funding: This work has been supported in part by Grant RTI2018-094336-B-I00 by the Spanish Government and the European Union Commission (MINECO/FEDER, UE). The second author is supported by UGC, New Delhi, India. The support is gratefully acknowledged. 
Acknowledgments: We gratefully acknowledge the suggestions of the learned referees.

Conflicts of Interest: The authors declare no conflict of interest.

\section{References}

1. Goebel, K.; Kirk, W.A. Topics in Metric Fixed Point Theory (Cambridge Studies in Advanced Mathematics); Cambridge University Press: Cambridge, UK, 1990.

2. Khamsi, M.A.; Kirk, W.A. An Introduction to Metric Spaces and Fixed Point Theory; John Wiley \& Sons: Hoboken, NJ, USA, 2001.

3. Kirk, W.A.; Sims, B. Handbook of Metric Fixed Point Theory; Springer: Dordrecht, The Netherlands, 2001.

4. Zadeh, L.A. Fuzzy sets. Inf. Control 1965, 8, 338-353. [CrossRef]

5. Kramosil, I.; Michalek, J. Fuzzy metric and statistical metric spaces. Kybernetica 1975, 11, 326-334.

6. George, A.; Veeramani, P. On some result in fuzzy metric space. Fuzzy Sets Syst. 1994, 64, 395-399. [CrossRef]

7. Choudhury, B.S.; Das, K.; Das, P. Coupled coincidence point results for compatible mappings in partially ordered fuzzy metric spaces. Fuzzy Sets Syst. 2013, 222, 84-97. [CrossRef]

8. Grabiec, M. Fixed points in fuzzy metric spaces. Fuzzy Sets Syst. 1988, 27, 385-389. [CrossRef]

9. Gregori, V.; Sapena, A. On fixed-point theorems in fuzzy metric spaces. Fuzzy Sets Syst. 2002, 125, $245-252$. [CrossRef]

10. Mihet, D. On fuzzy contractive mappings in fuzzy metric spaces. Fuzzy Sets Syst. 2007, 158, 915-921. [CrossRef]

11. Saha, P.; Choudhury, B.S.; Das, P. A new contractive mapping principle in fuzzy metric spaces. Bull. dell'Uni. Math. Ital. 2016, 8, 287-296. [CrossRef]

12. Kaufman, A.; Gupta, M.M. Fuzzy Mathematical Models in Engineering Management Science; Elsevier Science Inc.: New York, NY, USA, 1988.

13. Bari, C.D.; Suzuki, T.; Vetro, C. Best proximity points for cyclic Mier-Keeler contractions. Nonlinear Anal. 2008, 69, 3790-3794. [CrossRef]

14. Choudhury, B.S.; Maity, P.; Sadarangani, K.B. A best proximity point theorem using discontinuous functions. J. Convex Anal. 2017, 24, 41-53.

15. Jleli, M.; Samet, B. Best proximity points for $\alpha-\psi$-proximal contractive type mappings and applications. Bull. Sci. Math. 2013, 137, 977-995. [CrossRef]

16. Jleli, M.; Karapinar, E.; Samet, B. Best proximity point result for MK-proximal contractions. Abstr. Appl. Anal. 2012, 2012, 193085. [CrossRef]

17. Karapinar, E. Best proximity points of cyclic mappings. Appl. Math. Lett. 2012, 25, 1761-1766. [CrossRef]

18. Raj, V.S. Best proximity point theorems for non-self mappings. Fixed Point Theory 2013, 14, 447-454.

19. Raj, V.S. A best proximity point theorem for weakly contractive non-self-mappings. Nonlinear Anal. 2011, 74, 4804-4808.

20. De la Sen, M.; Abbas, M.; Saleem, N. On Optimal Fuzzy best proximity coincidence points of proximal contractions involving cyclic mappings in non-Archimedean fuzzy metric spaces. Mathematics 2017, 5, 22. [CrossRef]

21. Saha, P.; Guria, S.; Choudhury, B.S. Determining fuzzy distance through non-self fuzzy contractions. Yugoslav J. Oper. Res. 2019. [CrossRef]

22. Shayanpour, H.; Nematizadeh, A. Some results on common best proximity point in fuzzy metric spaces. Bol. Soc. Paran. Mat. 2017, 35, 177-194. [CrossRef]

23. Vetro, C.; Salimi, P. Best proximity point results in non-Archimedean fuzzy metric spaces. Fuzzy Inf. Eng. 2013, 4, 417-429. [CrossRef]

24. Anastassiou, G.A. Fuzzy Mathematics: Approximation Theory; Springer: Berlin, Germany, 2010.

25. Gregori, V.; Minana, J.J.; Morillas, S. Some questions in fuzzy metric spaces. Fuzzy Sets Syst. 2012, 204, 71-85. [CrossRef]

26. Alber, Y.I.; Guerre-Delabriere, S. Principle of weakly contractive maps in Hilbert spaces. New Results Oper. Theory Its Appl. 1997, 98, 7-22.

27. Rhoades, B.E. Some theorems on weakly contractive maps. Nonlinear Anal. TMA 2001, 47, $2683-2693$. [CrossRef] 
28. Choudhury, B.S.; Konar, P.; Rhoades, B.E.; Metiya, N. Fixed point theorems for generalized weakly contractive mappings. Nonlinear Anal. Theory Methods Appl. 2011, 74, 2116-2126. [CrossRef]

29. Dorić, D. Common fixed point for generalized $(\psi, \phi)$-weak contractions. Appl. Math. Lett. 2009, 22, 1896-1900. [CrossRef]

30. Zhang, Q.; Song, Y. Fixed point theory for generalized $\phi$-weak contractions. Appl. Math. Lett. 2009, 22, 75-78. [CrossRef]

31. Saha, P.; Choudhury, B.S.; Das, P. A weak contraction in a fuzzy metric spaces. J. Uncertain Syst. in press.

32. Rodríguez López, J.; Romaguera, S. The Hausdorff fuzzy metric on compact sets. Fuzzy Sets Syst. 2004, 147, 273-283. [CrossRef]

33. Raza, Z.; Saleem, N.; Abbas, M. Optimal coincidence points of proximal quasi-contraction mappings in non-Archimedean fuzzy metric spaces. J. Nonlinear Sci. Appl. 2016, 9, 3787-3801. [CrossRef]

34. Komal, S.; Kumam, P.; Khammahawong, K.; Sitthithakerngkiet, K. Best proximity coincidence point theorems for generalized nonlinear, contraction mappings. Filomat 2018, 32, 6753-6768. [CrossRef]

35. Boothby, W. An Introduction to Differentiable Manifolds and Riemannian Geometry ; Academic Press: New York, NY, USA, 1975.

36. Goldstein, H. Classical Mechanics; Addison-Wesley: Boston, MA, USA, 1951.

37. Chiang, A.C. Elements of Dynamic Optimization; McGraw-Hill: New York, NY, USA, 1992.

(C) 2019 by the authors. Licensee MDPI, Basel, Switzerland. This article is an open access article distributed under the terms and conditions of the Creative Commons Attribution (CC BY) license (http://creativecommons.org/licenses/by/4.0/). 